\title{
Criptococosis meníngea y lupus eritematoso sistémico: Reporte de un caso y revisión de la literatura
}

\author{
Antonio G. Tristano*
}

\section{Cryptococcal meningitis and systemic lupus erythematosus: \\ A case report and review}

Cryptococcal meningitis is an uncommon but well-known and frequently fatal complication of systemic lupus erythematosus (SLE). The clinical manifestations are unspecific and frequently are confused with lupus activity. A patient with cryptococcal meningitis and SLE, who responded well to amphotericin deoxycolate therapy is reported. In a review through MEDLINE of the Spanish and English literature 57 cases of SLE and cryptococcal meningitis were found. Simultaneous presentation of both diseases has been reported in only 3 cases. This temporal coexistence suggests that specific underlying immune defects associated with SLE directly predisposes to mycotic infections.

Key words: Amphotericin B deoxycolate, cryptococcal meningitis, Cryptococcus neoformans, steroids, systemic lupus erythematosus, meningitis.

Palabras clave: Anfotericina B deoxicolato, criptococosis meníngea, Cryptococcus neoformans, corticoesteroides, lupus eritematoso sistémico, meningitis.

\section{Introducción}

$\mathrm{E}$ ntre 30 y $50 \%$ de la morbilidad y mortalidad de los pacientes con lupus eritematoso sistémico (LES) son atribuidas a las infecciones, principalmente de la piel, pulmones y sistema genitourinario. Las infecciones del sistema nervioso central (SNC) solamente constituyen el $3 \%$ de todas las infecciones ${ }^{1-3}$.

Los pacientes con LES son susceptibles a contraer infecciones micóticas profundas, principalmente relacionadas a la terapia corticoesteroidal. Las más frecuentes son la criptococcosis y la histoplasmosis, pero también se han reportado casos de aspergilosis y coccidioidomicosis, todas las cuales responden apropiadamente tanto a anfotericina B como a 5-fluocitosina ${ }^{4}$. La meningitis por Cryptococcus. neoformans es inusual, pero una reconocida complicación y fatal en los pacientes con LES, quienes en la mayoría de los casos desarrollan esta infección oportunista como resultado de la terapia inmunosupresora ${ }^{5}$.

Aquí se reporta el caso de una paciente con diagnóstico de LES y criptococcosis meníngea y se realiza una revisión de la literatura de los casos reportados en inglés y español a través de MEDLINE.

\section{Caso clínico}

Mujer de 17 años que padecía de LES. En el momento del diagnóstico debutó con una nefritis lúpica manifestada por un síndrome nefrótico, con proteínas en orina de 24 horas de $3,3 \mathrm{~g}$, colesterolemia de $280 \mathrm{mg} \%$ y albuminemia de $1,8 \mathrm{~g} / \mathrm{dL}$, por lo cual recibió tratamiento con metilprednisolona succinato en bolos durante tres días (tres dosis), y se mantuvo con prednisona $1 \mathrm{mg} / \mathrm{kg}$ de peso/día, observándose mejoría del cuadro. Igualmente la paciente presentó una cefalea intensa que calmaba parcialmente con analgésicos y sin signos de irritación meníngea, que fue interpretada como un síntoma asociado al lupus.

Se mantuvo hospitalizada para realizar la biopsia renal, pero la paciente abandonó el hospital contraviniendo la opinión médica y continuó el tratamiento con prednisona hasta 15 días después de su egreso, cuando motu proprio disminuyó a una dosis de $35 \mathrm{mg} /$ día.

Un mes después, la paciente acudió nuevamente al hospital para control y aceptó internarse para someterse a la biopsia renal.

Los exámenes demostraron deterioro de la función renal, asociado a un síndrome nefrótico impuro, con hipertensión arterial (160/110). Se aumentó la dosis de prednisona a $1 \mathrm{mg} / \mathrm{kg}$ de peso/día y se agregó captopril $50 \mathrm{mg}$ cada 8 horas. La paciente refería persistencia de la cefalea frontoparietal, opresiva, de fuerte intensidad, que ya no mejoraba con analgésicos y que era exacerbada por los movimientos de la cabeza, sin otros signos y síntomas asociados. Se sospechó una vasculitis del SNC. A los pocos días del ingreso presentó fiebre de $40^{\circ} \mathrm{C}$, náuseas, vómitos y fotofobia. Se le realizó una punción lumbar, obteniéndose LCR a una presión inicial de $37 \mathrm{~cm} \mathrm{H}_{2} \mathrm{O}$
Caracas, Venezuela Hospital Dr. Domingo Luciani. Servicio de Medicina Interna.

*Afiliación actual: Centro Médico Docente La Trinidad, Caracas, Venezuela.

Recibido: 2 de de marzo de 2009 Aceptado: 15 de noviembre de 2009

Correspondencia a: Antonio G Tristano mjtristano@cantv.net 
y final de $16 \mathrm{~cm} \mathrm{H}_{2} \mathrm{O}$, de aspecto turbio, incoloro, con 0 cél $/ \mathrm{mm}^{3}$, glucorraquia $5 \mathrm{mg} \%$, proteinorraquia 76,4 $\mathrm{mg} \%$, Pandy 2++. La tinción de Gram directa evidenció blastosporas abundantes y la coloración con tinta china fue positiva para levaduras encapsuladas. Tanto el cultivo de LCR como el hemocultivo fueron positivos para $C$. neoformans. Se suspendió el tratamiento con corticoesteroides y se inició terapia con anfotericina $\mathrm{B}$ deoxicolato en una dosis de $0,7 \mathrm{mg} / \mathrm{kg}$ de peso/día. Alcanzada una dosis acumulada de 1 gramo, se observó su recuperación completa. Luego de obtener un cultivo negativo del LCR para hongos se inició tratamiento con ciclofosfamida i.v. (500 a $1.000 \mathrm{mg}$ mensual) y corticoesteroides a bajas dosis, para el manejo de su nefropatía.

\section{Discusión}

La criptococcosis es una infección que aparentemente afecta tanto a individuos inmunocompetentes como inmunocomprometidos; de estos últimos, los más susceptibles son aquellos pacientes con deficiencias de linfocitos T. Es generalmente aceptado que la infección se adquiere por vía aérea y, una vez depositado en los pulmones, a través de la vía hematógena produce infección en otros tejidos, principalmente el cerebro ${ }^{4}$.

Numerosos estudios han demostrado que la terapia corticoesteroidal (prednisona $20 \mathrm{mg}$ diarios o su equivalente) incrementa la susceptibilidad a las infecciones a través de su efectos sobre la inmunidad celular ${ }^{3,6}$. Adicionalmente los corticoesteroides inhiben el reclutamiento de neutrófilos y monocitos-macrófagos al sitio de la inflamación y deprimen la actividad bactericida de los monocitos y neutrófilos. Sin embargo, antes de iniciarse su uso regular en pacientes con LES, se reportaban infecciones hasta en $40 \%$ de estos pacientes, atribuyéndolo a las anormalidades inmunológicas intrínsecas de la enfermedad ${ }^{7}$.

Datos recientes han sugerido que una variante específica de genes que codifican una proteína llamada lectina que une manosa (MBL del inglés mannose-binding lectin) y receptores Fcg podrían aumentar el riesgo de infecciones oportunistas en pacientes con $\mathrm{LES}^{8,9}$. MBL es una proteína sérica que juega un rol importante en la defensa inmunológica, particularmente durante la fase de contacto primaria entre el patógeno y el organismo. Ciertos polimorfismos genéticos que producen niveles bajos o deficiencias de MBL están asociados con un incremento en la susceptibilidad para infecciones y para el riesgo de desarrollar LES $^{10,11}$. Sin embargo, niveles bajos de MBL pueden ser encontrados en 4 a 10\% de la población adulta sana. Garred y cols ${ }^{12}$, reportaron una incidencia de infecciones cuatro veces mayor en pacientes con LES que fueron homocigóticos para la variante alélica del MBL. Igualmente, polimorfismos genéticos que afectan la afini- dad de los receptores Fc para unir las inmunoglobulinas podrían aumentar el riesgo de infección. Yee y cols ${ }^{13}$, encontraron que en 5 pacientes con LES e infección por Streptococcus pneumoniae, 4 eran homocigóticos para el alelo RI3I del FCgRIIA. Este polimorfismo resultó en una baja afinidad para unir la IgG2, una subclase de la IgG específica para la cápsula bacteriana ${ }^{13}$.

Por otro lado, se ha reportado que los pacientes con LES tienen anormalidades en la inmunidad mediada por células. Dado que la resistencia a la infección por $C$. neoformans depende principalmente de la inmunidad mediada por células incluyendo células CD4, linfocitos citotóxicos, natural killer, macrófagos activados, y citoquinas como interleuquina 12 (IL-12) y $\gamma$ interferón, los defectos inmunológicos intrínsecos observados en pacientes con LES los tornan susceptibles a infecciones oportunistas por hongos, incluso si estos pacientes no están recibiendo agentes inmunosupresores ${ }^{14}$.

Mientras Sieving y cols ${ }^{4}$, comunicó 33 paciente con LES e infección profunda por hongos, todos relacionados al tratamiento corticoesteroidal, Hung y $\operatorname{cols}^{14}$, Huston y cols ${ }^{15}$ y Mok y cols ${ }^{5}$, describieron pacientes con LES e infección del SNC por C. neoformans, simultánea al momento de diagnosticarse el LES.

Adicionalmente se ha sugerido que el compromiso renal, como sucedió en el caso presente, se asocia con una frecuencia mayor de infecciones más allá del grado atribuible a la dosis empleada de corticoesteroides ${ }^{16}$.

El compromiso del SNC es la mayor causa de morbilidad y mortalidad en los pacientes con LES; no obstante, la infección del SNC se considera una complicación inhabitual. En un estudio reciente de 3.165 pacientes con LES, se probó infección del SNC en 17 pacientes; en 59\% se identificó C. neoformans, en $23 \%$ Listeria monoytogenes, en $6 \%$ Enterobacter aerogenes y en $12 \%$ S. pneumoniae ${ }^{14}$.

La criptococcosis meníngea es una de las principales causas de infección del SNC en pacientes con LES y generalmente se presenta con un cuadro clínico inespecífico, que puede confundirse con manifestaciones de actividad lúpica del SNC (vasculitis) o ser enteramente asintomática. Las manifestaciones clínicas más frecuentes son fiebre y cefalea, seguidas de náuseas, vómitos, convulsiones, trastornos del estado de conciencia, rigidez de nuca y diplopía, entre otras ${ }^{14,16,17}$.

El análisis citoquímico del LCR arroja hallazgos semejantes a los observados en la tuberculosis o la meningitis viral. El examen microscópico es positivo entre 50 y $60 \%$ de los casos. Sin embargo, aunque el estudio del LCR muestre un recuento de leucocitos normal, no debe descartarse el diagnóstico de criptococcosis meníngea en pacientes con LES, por lo que las preparaciones con tinta china son necesarias para completar el estudio diagnóstico ${ }^{18}$. De los nueve pacientes con LES y criptococcosis meníngea reportados por Hung y cols ${ }^{14}, 4$ de ellos 
mostraron recuento de leucocitos normal en el análisis del LCR. En casos donde el estudio microscópico sea negativo, el cultivo en el medio de Sabouraud puede dar el diagnóstico en 2 a 4 días. En conjunción se pueden utilizar pruebas serológicas ${ }^{18}$. Una prueba serológica útil, es la aglutinación en látex para el antígeno de C. neoformans, el cual detecta concentraciones tan bajas como 25-60 ng del antígeno por $\mathrm{ml}$ de fluido del cuerpo y puede ser positivo cuando el cultivo y la tinta china son negativos ${ }^{3}$.

El tratamiento se basa en la utilización de anfotericina B la que mejora a $\sim 80 \%$ de los pacientes, aunque es esperable hasta $30 \%$ de recaídas ${ }^{17,18}$. La dosis promedio recomendada es de $1 \mathrm{mg} / \mathrm{kg} /$ día, hasta acumular entre $1 \mathrm{y}$ $1,5 \mathrm{gr}^{18}$. El otro medicamento utilizado es 5-fluocitosina

Tabla 1. Criptococcosis meníngea en pacientes con lupus eritematoso sistémico (LES): Casos reportados en la literatura

\begin{tabular}{|c|c|c|c|c|c|}
\hline Autor & $\begin{array}{c}\text { Casos } \\
\text { (n) }\end{array}$ & Medicamentos previos a la infección & Tratamiento & Evolución & Ref. \\
\hline Sieving et al & 2 & Corticoesteroides, azatioprina & A & Muerte & 4 \\
\hline Mok et al & 1 & Ninguno & $\mathrm{A}, \mathrm{FC}, \mathrm{FL}$ & Recuperación & 5 \\
\hline Hung et al & 9 & 8 con corticoesteroides, 1 ninguno & $\mathrm{A}, \mathrm{FL}, \mathrm{FC}$ & 5 muertes, 4 recuperación & 14 \\
\hline Huston et al & 1 & Ninguno & $A, F L$ & Recuperación & 15 \\
\hline Coto et al & 10 & Corticoesteroides, azatriopina, Metotrexato, ciclofosfamida & $A, F C$ & 2 muertes, 3 complicados, 5 recuperación & 16 \\
\hline Zimmermann et al & 2 & Corticoesteroides & A, FC & Recuperación & 17 \\
\hline Harvey et al* & 1 & Ninguno & Ninguno & Muerte & 17 \\
\hline Rapaport et al* & 2 & Corticoesteroides & Ninguno & Muerte & 17 \\
\hline Pariser et al* & 1 & Corticoesteroides & Ninguno & Muerte & 17 \\
\hline $\mathrm{MGH}^{*}$ & 1 & Ninguno & Ninguno & Muerte & 17 \\
\hline Collins et al* & 2 & Corticoesteroides, azatioprina & 1 Ninguno, A & Muerte, recuperación & 17 \\
\hline Cooke et al* & 1 & Corticoesteroides & Ninguno & Muerte & 17 \\
\hline Khan et al* & 1 & Corticoesterorides & A & Recuperación & 17 \\
\hline Roberts et al* & 1 & Corticoesteroides, azatioprina & $A, F C$ & Recuperación & 17 \\
\hline Malas e al* & 1 & Corticoesteroides, azatioprina & $A, F C$ & Muerte & 17 \\
\hline Lesser et al* & 1 & Corticoesteroides, azatioprina & $A$ & Recuperación & 17 \\
\hline Eiser et al* & 1 & Corticoesteroides, azatioprina, ciclofosfamida & A & Recuperación & 17 \\
\hline Perfect et al* & 2 & Corticoesteroides, ciclofosfamida & $A, F C$ & Muerte, recuperación & 17 \\
\hline Smith et al* & 1 & Corticoesteroides, azatioprina & A & Recuperación & 17 \\
\hline Li et al* & 1 & Corticoesteroides, azatioprina & A, FC, miconazol & Recuperación con ceguera & 17 \\
\hline Kong et al* & 3 & Corticoesteroides, azatioprina & $A, F C$ & 2 muertes, recuperación & 17 \\
\hline Al-Rasheed et al* & 1 & Corticoesteroides & $A, F C$ & Muerte & 17 \\
\hline Speller et al & 2 & Corticoesteroides, azatioprina & $A, F C$ & Muerte, recuperación & 19 \\
\hline Liou et al & 1 & Corticoesteroides, ciclofosfamida, hidroxicloroquina & $\mathrm{A}, \mathrm{FL}$ & Recuperación & 20 \\
\hline Prasad et al & 1 & No reportado & $A$ & Recuperación & 21 \\
\hline Ferreira et al & 1 & Corticoesteroides & $\mathrm{FL}$ & Recuperación & 22 \\
\hline Wong et al & 2 & Corticoesteroides, ciclofosfamida, azatioprina & $A, F C$ & Recuperación & 23 \\
\hline Hiruma et al & 1 & Corticoesteroides & $A, F C$ & Recuperación & 24 \\
\hline Akcaglar et al & 1 & Corticoesteroides & A & Muerte & 25 \\
\hline Suzuki et al & 1 & Corticoesteroides & $\mathrm{FL}$ & Muerte & 26 \\
\hline Kwok et al & 1 & Corticoesteroides, ciclofosfamida, M, azatioprina & $A, F C$ & Recuperación & 27 \\
\hline
\end{tabular}


la cual es mejor tolerada, menos tóxica y penetra mejor en el SNC; se describe, sin embargo, la emergencia de resistencia durante el tratamiento ${ }^{19}$. Se recomienda la utilización de ambos medicamentos en combinación, para luego seguir con fluconazol. La óptima duración del tratamiento no ha sido determinada y dependerá de los resultados de las repetidas punciones lumbares o de las pruebas serológicas. Anfotericina B podría prevenir la multiplicación de hongos resistentes a 5-fluocitosina, mientras que las altas dosis iniciales de 5-fluocitosina, producen una actividad anticriptococcósica sin efectos tóxicos mientras la anfotericina $\mathrm{B}$ aumenta gradualmente su actividad en el transcurso de los días; además se ha reportado efectos sinérgicos entre los dos medicamentos ${ }^{19}$.

La mortalidad alcanza hasta 54\% y la mayoría de los fallecimientos ocurren en las primeras semanas de la infección ${ }^{14,17}$. En la revisión de la literatura científica se encontró que han sido reportados 57 casos de pacientes con LES y criptococcosis meníngea, de los cuales junto con el reportado aquí, hay solamente 4 casos cuya infección no estuvo necesariamente relacionada al tratamiento inmunosupresor (Tabla 1).

\section{Conclusiones}

Se presenta un caso de una paciente con LES, quien contrajo precozmente en su evolución una meningitis por
C. neoformans, que fue tratada satisfactoriamente con anfotericina B deoxicolato. Además de los corticoesteroides, los defectos inmunológicos subyacentes en los pacientes con LES contribuyen a su susceptibilidad a la infecciones. La criptococcosis meníngea en pacientes con LES es una complicación inusual pero bien reconocida y el diagnóstico precoz y el tratamiento antimicótico efectivo mejoran el pronóstico de estos pacientes.

\section{Resumen}

La meningitis por Cryptococcu neoformans es una inusual pero reconocida fatal complicación en los pacientes con lupus eritematoso sistémico (LES). Se presenta con un cuadro clínico inespecífico y frecuentemente se confunde con la actividad lúpica. Se reporta un caso de un paciente diagnosticado con meningitis por C. neoformans, al comienzo de LES, que fue tratada satisfactoriamente con anfotericina B deoxicolato. En la revisión de la literatura en español e inglés a través de MEDLINE, se encontró que han sido reportados 57 casos de pacientes con LES y criptococosis meníngea, de los cuales hay solamente 3 casos cuya infección se presentó simultáneamente con el LES. La presentación simultánea de criptococosis meníngea y LES enfatiza la posibilidad de que defectos inmunológicos intrínsecos en pacientes con LES sean los directamente responsables de la predisposición a adquirir infecciones micóticas.

\section{Referencias}

1.- Juárez M, Misischia R, Alarcón G S. Infection in systemic connective tissue diseases: systemic lupus erythematosus, scleroderma, and polymyositis / dermatomyositis. Rheum Dis Clin North Am 2003; 29: 163-84.

2.- Gladman D D, Hussain F, Ibáñez D, Urowitz M B. The nature and outcome of infection in systemic lupus erythematosus. Lupus 2002; 11: 234-9.

3.- Zonana-Nacach A, Camargo-Coronel A, Yañez P, Sánchez L, Jiménez-Balderas F J, Fraga A. Infections in outpatients with systemic lupus erythematosus: a prospective study. Lupus 2001; 10: 505-10.

4.- Sieving R R, Kauffman C A, Watanakunakorn C. Deep fungal infection in systemic lupus erythematosus-three cases reported, literature reviewed. J Rheumatol 1975; 2 (1): 61-72.

5.- Mok C C, Lau C S, Yuen K Y. Cryptococcal meningitis presenting concurrently with systemic lupus erythematosus. Clin Exp Rheumatol 1998; 16 (2): 169-71.
6.- Petri M, Genovese M. Incidence of and risk factors for hospitalizations in systemic lupus erythematosus: A prospective study of the Hopkins Lupus Cohort. J Rheumatol 1992; 19: $1559-65$

7.- Klemperer P, Pollack A D, Baehr G. Pathology of disseminated lupus erythematosus. Arch Pathol 1941; 32: 569.

8.- Holers V M. Complement deficiency states, disease susceptibility, and infection risk in systemic lupus erythematosus. Arthritis Rheum 1999; 42 (10): 2023-5.

9.- Fessler B J. Infectious diseases in systemic lupus erythematosus: risk factors, management and prophylaxis. Best Pract Res Clin Rheumatol 2002; 16 (2): 281-91. Review.

10.- Sullivan K E, Wooten C, Goldman D, Petri M. Mannose-binding protein genetic polymorphisms in black patients with systemic lupus erythematosus. Arthritis Rheum 1996; 39 (12): 2046-51.

11.- Garred P, Madsen H O, Halberg P, Petersen J, Kronborg G, Svejgaard A, et al. Mannose-binding lectin polymorphisms and susceptibility to infection in systemic lupus erythematosus. Arthritis Rheum 1999; 42 (10): 2145-52.

12.- Turner M W. Mannose-binding lectin (MBL) in health and disease. Immunobiol 1998; 199: 327-39.

13.- Yee A M, Ng S C, Sobel R E, Salmon J E. Fe gammaRIIA polymorphism as a risk factor for invasive pneumococcal infections in systemic lupus erythematosus. Arthritis Rheum 1997; 40 (6): 1180-2.

14.- Hung J J, Ou L S, Lee W I, Huang J L. Central nervous system infections in patients with systemic lupus erythematosus. J Rheumatol 2005; 32 (1): 40-3.

15.- Huston K K, Gelber A C. Simultaneous presentation of cryptococcal meningitis and lupus nephritis. J Rheumatol 2005; 32 (12): 2501-2.

16.- Coto D J, Campos A R. Central nervous system cryptococcosis in 10 patients with systemic lupus erithematosus. Rev Clin Esp 1995; 195 (1): $12-5$.

17.- Zimmermann B, Spiegel M, Lally E V. Cryptococcal meningitis in systemic lupus erythematosus. Semin Arthritis Rheum 1992; 22 (1): 18-24. 
18.- Wilson T S, Fleming W A, Robinson F L, Nicholl B. Cryptococcal meningitis associated with steroid therapy. J Clin Path 1970; 23: 657-63.

19.- Speller D C, Fakunle F, Cairns S A, Stephens M. Cryptococcal meningitis complicating systemic lupus erythematosus; two patients treated with flucytosine and amphotericin B. J Clin Path 1977; 30: 254-61.

20.- Liou J, Chiu C, Tseng C, Chi C, Fu L. Cryptococcal meningitis in pediatric systemic lupus erythematosus. Mycoses 2003; 46 (3-4): 153-6.

21.- Prasad K N, Agarwal J, Nag V L, Verma A K, Dixit A K, Ayyagari A. Cryptococcal infection in patients with clinically diagnosed meningitis in a tertiary care center. Neurol India 2003; 51 (3): 364-6.

22.- Ferreira R C, Phan G, Bateman J B. Favorable visual outcome in cryptococcal meningitis. Am J Ophthalmol 1997; 124 (4): 558-60.

23.- Wong K L, Woo E K, Yu Y L, Wong R W. Neurological manifestations of systemic lupus erythematosus: a prospective study. Q J Med 1991; 81 (294): 857-70.

24.- Hiruma M, Kagawa S. Ultrastructure of Cryptococcus neoformans in the cerebrospinal fluid of a patient with cryptococcal meningitis. Mycopathol 1985; 89(1): 5-12.
25.- Akcaglar S, Sevgican E, Akalin H, Ener B, Tore $\mathrm{O}$. Two cases of cryptococcal meningitis in immunocompromised patients not infected with HIV. Mycoses 2007; 50 (3): 235-8.

26.- Suzuki K, Nakase K, Ino K, Sugawara Y, Sekine T, Katayama N. Breakthrough cryptococcosis in a patient with systemic lupus erythematosus (SLE) receiving micafungin. J Infect Chemother 2008; 14: 311-4.

27.- Kwok S-K, Seo S-H, Ju JH, Yoon C-H, Park S C, Kim B S, et al. Cryptococcal meningitis presenting with isolated sixth cranial nerve palsy in a patient with systemic lupus erythematosus. J Korean Med Sci 2008; 23: 153-5.

Nota del Editor: Con posterioridad a la aceptación de este artículo se ha publicado una guía clínica cuya lectura recomendamos a los lectores. John R. Perfect, William E. Dismukes, Francoise Dromer, David L. Goldman, John R. Graybill, Richard J. Hamill et al, en representación de IDSA. Clinical Practice Guidelines for the Management of Cryptococcal Disease: 2010 Update by the Infectious Diseases Society of America. Clin Infect Dis 2010; Feb 1; 50: 291-322. 